\title{
Clostridium difficile: An Important Opportunistic Pathogen in Healthcare-Associated Infections ${ }^{1}$
}

\author{
Soohyoun Ahn and Amarat H. Simonne ${ }^{2}$
}

Healthcare-associated infections (HAIs) are defined as infections that patients can get while receiving medical treatment in a healthcare facility. HAIs have become a major threat to the safety of patients who often have weakened immune systems, such as people with HIV/AIDS or those on immune-suppressing therapies. One opportunistic pathogen, Clostridium difficile, has been getting more attention in recent years because of its association with antibiotic use and high death rate among the elderly. This factsheet will provide an overview of Clostridium difficile infection (CDI) with a focus on healthcare-associated infections.

\section{What are healthcare-associated infections?}

Healthcare-associated infections (HAIs) are infections that patients acquire during the course of receiving healthcare treatment for medical or surgical conditions. These infections related to medical care can be devastating to human health and are sometimes deadly. In 2002, the estimated number of HAIs in US hospitals, adjusted to include federal facilities, was approximately 1.7 million (Klevens et al. 2007). According to the HAI prevalence survey published in 2014, there were an estimated 721,800 HAIs in acute care hospitals in the United States in 2011 (Table 1) (Magill et al. 2014). Additionally, approximately 75,000 patients with HAIs died during their hospitalizations (CDC 2016a). The overall annual direct medical costs of HAI to US hospitals ranges from $\$ 28.4$ to $\$ 33.8$ billion (Scott 2009).

Traditionally, HAIs have been commonly associated with the insertion of medical devices like catheters and ventilators, which include central line-associated bloodstream infections from tubes placed into patients' veins to draw blood or give medications, catheter-associated urinary tract infections, and ventilator-associated pneumonia. Consequently, device-associated infections have been a major focus of HAI prevention in recent decades. According to the recent survey study (Magill et al. 2014), deviceassociated infections account for only $25.6 \%$ of all HAIs; however, infections not associated with devices or operative procedures, including C. difficile infections, account for approximately $50 \%$ of all HAIs. Therefore, surveillance and prevention of $C$. difficile infections has become a recent focus of the Centers for Disease Control and Prevention (CDC).

\section{What are opportunistic pathogens?}

Opportunistic pathogens are microorganisms that are normally associated with different parts of the human body. They usually do not cause disease in healthy individuals, but have the ability to cause disease under certain conditions, such as weakened immunity. Opportunistic pathogens are different from other pathogens that commonly

1. This document is FSHN14-07, one of a series of the Food Science and Human Nutrition Department, UF/IFAS Extension. Original publication date September 2014. Revised January 2018. Visit the EDIS website at http://edis.ifas.ufl.edu.

2. Soohyoun Ahn, assistant professor, Food Science and Human Nutrition Department; and Amarat H. Simonne, professor, Department of Family, Youth and Community Sciences; UF/IFAS Extension, Gainesville, FL 32611.

The Institute of Food and Agricultural Sciences (IFAS) is an Equal Opportunity Institution authorized to provide research, educational information and other services only to individuals and institutions that function with non-discrimination with respect to race, creed, color, religion, age, disability, sex, sexual orientation, marital status, national origin, political opinions or affiliations. For more information on obtaining other UF/IFAS Extension publications, contact your county's UF/IFAS Extension office. 
cause disease, as they are usually benign and frequently associated with normal human body functions. Usually, the human body gets along well with these opportunistic pathogens and, in some cases, even depends on them. However, sometimes things can go wrong, and a good relationship can turn bad. For example, E. coli plays an important role in degrading organic matter in the intestine and providing key nutrients to the body, but under certain circumstances, E. coli can migrate into the urinary tract or bladder and cause infection. When opportunistic pathogens cause infections, they are called opportunistic infections.

Different kinds of opportunistic pathogens have been known to humans for many years. Candida albicans, a normal inhabitant of the mucous membranes of the oral cavity and genitourinary tract, can cause an oral disease called oral thrush in people who have a compromised immune system or who are taking antibiotics. E. coli normally inhabits the intestine, but it may cause infection when transferred to other body regions. Oral Streptococcus species that enter the bloodstream during oral surgery have been linked to heart disease (Banas 2004). Pseudomonas aeruginosa, found on vegetables and in soil, is one of the most prevalent causes of infections on burned skin and is a significant cause of pneumonia in long-term hospital patients (Tredget et al. 2004; Chroneou et al. 2007; Kerr and Snelling 2009; Bassetti et al. 2014). Additionally, some bacteria are known to cause serious infections in people who have received services in healthcare facilities. These include Clostridium difficile, Staphylococcus aureus, Acinetobacter, and Klebsiella spp. (CDC 2014).

\section{When do opportunistic pathogens cause infection?}

Opportunistic infections usually occur when there are changes in the overall status of the body. We become susceptible to opportunistic infections when we develop another disease or injury (e.g. burn) or after artificial manipulations, such as use of catheter (Pagano and Caira 2012; Dabziger-Isakov 2014; Kyi et al. 2014). When we undergo antibiotic treatment, it can disrupt the balance of normal microorganisms in the body and it can lead to opportunistic infection. In addition, when our immune systems become compromised, normal microflora can overpopulate or move into areas of the body where they do not normally occur, which can result in opportunistic infection.

\section{What is Clostridium difficile?}

Clostridium difficile (pronounced as klos-TRID-e-um difuh-SEEL), also known as C. diff for short, is an anaerobic, gram-positive bacterium. It is a very common environmental bacterium that is found in water, air, soil, and many other environments. However, it is best known for causing antibiotic-associated diarrhea (Knoop et al. 1993) or as a cause of infection associated with healthcare settings, such as hospitals and nursing homes (Lessa et al 2015). Usually, C. difficile is a part of normal colonic microflora, but when competing bacteria in the gut are wiped out by antibiotic treatment, $C$. difficile can cause diarrheal disease and, in severe cases, life-threatening inflammation of the colon called toxic megacolon (Hookman and Barkin 2009; Norén 2010). In this rare condition of toxic megacolon, your colon is unable to expel gas and stool, causing it to become greatly distended (megacolon). Left untreated, your colon may rupture, causing bacteria from the colon to enter your abdominal cavity. An enlarged or ruptured colon requires emergency surgery and may be fatal.

C. difficile can form spores when environmental conditions cannot support its continued growth. This spore-forming ability allows this organism to persist in the environment, because spores can survive in harsh environmental conditions for months. It can live in soil or even on dry surfaces for extended periods of time. Due to the spore-forming ability, it is extremely hard to eliminate $C$. difficile; therefore, it has become an increasing problem for healthcare settings.

\section{Why is Clostridium difficile important?}

Clostridium difficile is the most frequently reported agent for HAIs, accounting for $12.1 \%$ of all HAIs (Table 2) (Magill et al 2014; Lessa et al 2015; Napolitano et al 2017). It is estimated that $C$. difficile infection (CDI) causes over 450,000 illnesses each year in the United States, and the majority of these infections are hospital-acquired (Kuehn 2011; Lessa et al. 2015; CDC 2016b). While most types of HAIs are declining, infection caused by $C$. difficile remains at historically high levels. According to a CDC report, the number of deaths due to C. difficile enterocolitis, an inflammation of the intestines, substantially increased from 793 deaths in 1999 to 8,085 deaths in 2011 (Kochanek et al. 2016). CDI is currently estimated to cause 29,000 deaths in the United States every year, with an approximate economic burden of $\$ 4.8$ billion (CDC 2015; 2016b). In the 2016 National Vital Statistics Reports, which lists causes of death and death rates in 2014, CDI ranked as the $18^{\text {th }}$ leading 
cause of death for the population aged 65 and older, and nearly $90 \%$ of deaths associated with CDI occurred among people aged 65 and over (Kochanek et al 2016). In addition, a report from the US Department of Health and Human Services shows that the national rate of CDI-related hospitalizations has continually increased over the past decade, from 8.8 per 1,000 adult discharges in 2004 to 14.7 in 2015, with a projection of continuous increases for coming years (Steiner et al. 2015; Florida Department of Health 2015). The Florida Department of Health's Division of Disease Control and Health Protection published a report analyzing CDI statistics from 2008 to 2013 (Teter 2015). According to this report, the total CDI prevalence in Florida remained below national estimates; however, it remained above the HAI Prevention Target established by the US Department of Health and Human Services (DHHS) for the entire period (Figure 1). Additionally, in Florida, emergency admissions showed a significantly higher prevalence than urgent or elective admission types across this six-year period (Figure 2).



Figure 1. Prevalence of $C D I$ in hospital discharges in Florida. While total CDI prevalence in Florida remained below national estimates, it was above the HAI Prevention Target established by the Department of Health and Human Services (DHHS). This figure was created based on the data from a report by Florida Department of Health (Teter 2015).

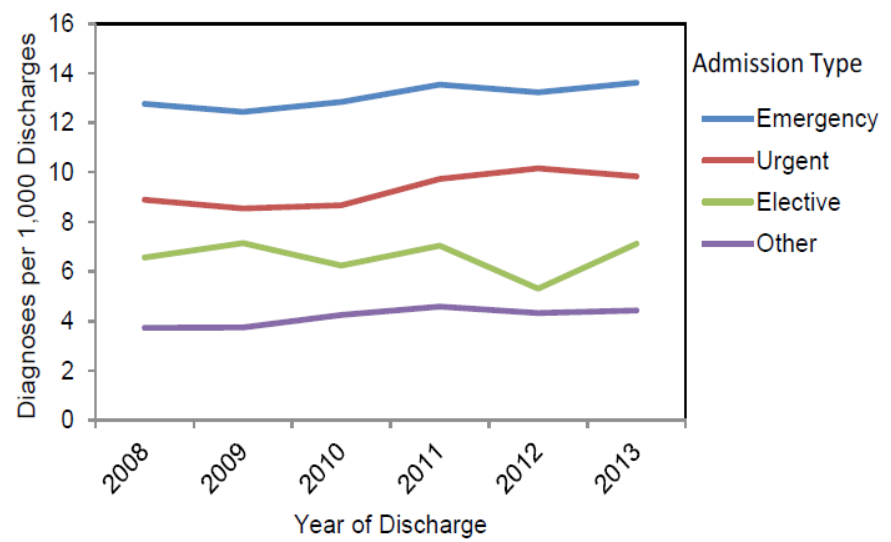

Figure 2. Overall CDI discharge prevalence in Florida by admission type from 2008 to 2013. Emergency admissions had a significantly higher prevalence (range: 12.43-13.61 per 1,000 discharges) than other admission types (Teter 2015).

\section{What are the symptoms of Clostridium difficile infection (CDI)?}

Symptoms of CDI range from self-limited diarrhea to a combination of symptoms. Most common symptoms are watery diarrhea, having at least three bowel movements per day for two or more days, fever, loss of appetite, nausea, and abdominal pain and/or tenderness. When the disease worsens, patients can progress to kidney failure, fever, elevated white blood cell count, and tachycardia (abnormally rapid heart rate), which may require admission to intensive care unit. However, many patients with CDI remain asymptomatic after infection, but can shed C. difficile in the stool.

\section{How is CDI transmitted to humans?}

Clostridium difficile is commonly found in the environment, including soil, air, and water. C. difficile is also shed in human and animal feces. Therefore, any surface, device, or material (e.g. toilets, bathing tubs, and electronic rectal thermometers) that become contaminated with feces may serve as a reservoir for $C$. difficile or its spores. A small number of healthy people can carry $C$. difficile in their intestine without showing any symptoms of CDI, and can spread $C$. difficile through fecal contamination. In healthcare settings, $C$. difficile and/or its spores are transferred to patients mainly via the hands of healthcare personnel who have come in contact with a contaminated surface or item. Spores produced by $C$. difficile can live for long periods on surfaces and may be found on bed linens, bed rails, bathroom fixtures, and medical equipment. Spores on these surfaces easily spread to patients, either directly or through the hands of doctors, nurses, and other healthcare 
providers, as well as visitors if they do not wash their hands thoroughly.

C. difficile transmission in community-acquired CDIs, which is not associated with healthcare setting, is not well documented; however, multiple recent studies have implicated companion pets and farm animals as a potential source of $C$. difficile transmission to humans (Loo et al. 2016; Stone et al. 2016).

\section{Who is at risk of CDI?}

Illness from CDI mostly affects the elderly in hospitals or in long-term care facilities. C. difficile infection also typically occurs after use of antibiotic treatment. If you have a serious illness or a weakened immune system due to medical treatment (e.g. chemotherapy) or need to use a feeding tube, you are more susceptible to CDI. Having gastrointestinal procedures or abdominal surgery also increases your risk of CDI. Older age is also a risk factor for CDI. It has been reported that the risk of CDI is 10 times greater for people of age 65 and older compared to younger people (Pépin et al. 2005; Leffler and Lamont 2015). However, studies show increasing rates of CDI among people traditionally not considered high risk, such as younger and healthy individuals without a history of antibiotic use or exposure to healthcare facilities.

\section{How is CDI diagnosed?}

Doctors often suspect CDI when patients have diarrhea lasting more than two days, have taken antibiotics within the past 2 months or so, or when patients develop diarrhea a few days after hospitalization. In these cases, CDI can be diagnosed by using one or more of the following tests:

1. Stool test: This is most commonly used diagnostic method. Toxin produced by $C$. difficile can be detected in a patient's stool sample through lab tests.

2. Imaging test: This test is usually used to confirm possible complications of $C$. difficile rather than diagnosis of CDI itself. Doctor can order an abdominal x-ray or a computerized tomography (CT) scan when he suspects CDI complications. This test can determine the presence of CDI complications such as colon wall thickening, bowel expansion, or a hole (perforation) in the lining of the colon.

3. Colon examination: CDI can also be diagnosed by examining the inside of colon. For this test (colonoscopy), a flexible tube with a small camera on one end is inserted into a patient's colon so a doctor can look for any inflammation.

\section{How can CDI be treated?}

Initially, CDI is managed by discontinuing antibiotics that have led to CDI if a patient is still taking it. C. difficile infection is generally treated for 10 days with antibiotics prescribed by a doctor, most commonly oral metronidazole or vancomycin (Kelly and Lamont 2014). This treatment is effective and has few side effects. In about $20-25 \%$ of patients, CDI can be resolved without any treatment simply by discontinuing the antibiotic the patient was taking within 2-3 days (Squellati 2017). In severe cases, however, a patient might need surgery to remove the infected part of the intestines. This type of surgery is rare and is needed in only small number of patients with CDI.

One problem with CDI treatment is a high recurrence rate associated with the disease. CDI returns in approximately $20 \%$ of patients either because C. difficile was not fully eliminated in initial infection or because patients were reinfected. In a small number of recurrence cases, the $\mathrm{CDI}$ can return two or more times with recurrence rate increasing up to $65 \%$ (CDC 2015). CDI recurrence is more common in older patients (age of 65 or older) or patients who are taking other antibiotics for different conditions while being treated with antibiotics for CDI or who have a severe underlying medical disorder such as chronic kidney failure or inflammatory bowel disease (IBD). Recurrent CDI can be treated with antibiotics (typically vancomycin or fidaxomicin). Alternatively, fecal microbiota transplantation (FMT), also known as a stool transplant, has been clinically tested with a success rate higher than $90 \%$ for treating CDI (Rohlke and Stollman 2012). FMT restores healthy intestinal bacteria by transplanting stool from a healthy donor to the colon of a patient. However, FMT has not been approved by the FDA and is mostly used in clinical trials only.

\section{How can CDI be prevented? Preventing CDI in Individuals}

Do not take antibiotics unless they have been prescribed by your healthcare provider. If you begin taking antibiotics, continue taking them as directed until you have finished all the pills, even if you feel better after just one or two days. If you don't finish your antibiotics, you risk growing antibiotic-resistant bacteria in your own gut. If you have trouble tolerating your antibiotics, call your healthcare provider. Practice good basic personal hygiene and wash your hands thoroughly and frequently (Dubberke and 
Gerding 2011). You should wash hands carefully with soap and warm water, especially after using the bathroom and before eating. Since $C$. difficile is often associated with healthcare-originated infection, your healthcare providers should clean their hands properly and frequently and use gloves appropriately.

\section{Healthcare Providers and Patient Visitors}

If you are a healthcare provider, or if you take care of or visit CDI patients, you should wear gloves and a gown whenever you visit, especially during the duration of the patient's diarrhea to prevent transmission of $C$. difficile to other people (Sehulster and Chinn 2003; Siegel et al. 2007). Remove gloves and gown immediately on leaving the patient's room and dispose of them appropriately (or follow established protocols for re-usable gowns). All healthcare providers, as well as a patient's family and visitors, should comply with hand hygiene recommendations. Rooms and items used by CDI patients must be adequately cleaned and disinfected with a freshly made $10 \%$ bleach solution. CDI patients should use a single room or share a room only with other CDI patients, and should remain confined to their rooms. They should not go to common areas except for treatments or tests. Because it is very difficult to completely eliminate all $C$. difficile spores, it should be assumed that C. difficile is present in healthcare settings. Therefore, most importantly, every person who enters the treatment area, including healthcare providers, housekeeping and administration staff, patients and their families, visitors, and volunteers, must be educated about CDI and how to prevent the spread of the infection in healthcare settings.

\section{References}

Banas, J. A. 2004. "Virulence properties of Streptococcus mutans." Front Biosci 9:1267-1277.

Bassetti, M., G. Villa, and D. Pecori. 2014. "Antibioticresistant Pseudomonas aeruginosa: focus on care in patients receiving assited ventilation." Future Microbiol 9:465-474.

Centers for Disease Control and Prevention (CDC). 2014. "Diseases and organisms in healthcare settings." Last accessed on November 6, 2017. http://www.cdc.gov/HAI/ organisms/organisms.html

Centers for Disease Control and Prevention (CDC). 2015. "Clostridium difficile infection information for patients." Last accessed on November 30, 2017. https://www.cdc.gov/ hai/organisms/cdiff/cdiff-patient.html
Centers for Disease Control and Prevention (CDC). 2016a. "HAI data and statistics." Last accessed on November 3, 2017. https://www.cdc.gov/hai/surveillance/index.html

Centers for Disease Control and Prevention (CDC). 2016b. "Clostridium difficile infection (CDI) - Prevention primer." Last accessed on November 14, 2017. https://www.cdc.gov/ hai/pdfs/toolkits/cdi-primer-2-2016.pdf

Chroneou, A., N. Zias, J. F. Beamis, Jr, and D. E. Craven. 2007. "Healthcare-associated pneumonia: principles and emerging concepts on management." Expert Opin Pharmacother. 8:3117-3131.

Danziger-Isakov, L. 2014. "Gastrointestinal infections after transplantation." Curr Opin Gastroenterol 30:40-46.

Dubberke, E. R., and D. N. Gerding. 2011. "Rationale for hand hygiene recommendations after caring for a patient with Clostridium difficile infection." Last accessed on November 30, 2017. https://www.shea-online.org/images/ patients/CDI-hand-hygiene-Update.pdf

Hookman, P., and J. S. Barkin. 2009. "Clostridium difficile associated infection, diarrhea and colitis." World J. Gastroenterol. 15:1554-1580.

Kelly, C. P., and J. T. Lamont. 2014. "Clostridium difficile in adults: treatment." Last accessed on November 30, 2017. http://www.uptodate.com/contents/ clostridium-difficile-in-adults-treatment

Kerr, K. G. and A. M. Snelling. 2009. "Pseudomonas aeruginosa: a formidable and ever-present adversary." J. Hosp. Infect. 73:338-344.

Klevens, R. M., J. R. Edwards, C. L. Richards, Jr., T. C. Horan, R. P. Gaynes, D. A. Pollock, and D. M. Cardo. 2002. "Estimating health care-associated infections and deaths in US hospitals. Public Health Rep. 122:160-166.

Knoop, F. C., M. Owens, and I. C. Crocker. 1993.

"Clostridium difficile: clinical disease and diagnosis." Clin. Microbiol. Rev. 6:251-265.

Kochanek, K. D., S. L. Murphy, J. Xu, and B. Tejada-Vera. 2016. "Deaths: final data for 2014." Natl Vital Stat Rep 65:1-122. Last accessed on November 30, 2017. https:// www.cdc.gov/nchs/data/nvsr/nvsr65/nvsr65_04.pdf

Kuehn, B. M. 2011. "Scientists seek strategies to prevent Clostridium difficile infections." JAMA 306:1849-1850. 
Kyi, C., M. D. Hellmann, J. D. Wolchok, P. B. Chapman, and M. A. Postow. 2014. "Opportunistic infections in patients treated with immunotherapy for cancer." J. Immunother Cancer. 2:19.

Leffler, D. A. and J. T. Lamont. 2015. "Clostridium difficile infection.” N. Engl. J. Med. 372:1539-1548.

Lessa, F. C., L. G. Winston, L. C. McDonald, and Emerging Infections Program C. difficile Surveillance Team. 2015. "Burden of Clostridium difficile infection in the United States.” N. Engl. J. Med. 372:2369-2370.

Loo, V. G., P. Brassard, and M. A. Miller. 2016. "Household transmission of Clostridium difficile to family members and domestic pets." Infect. Control Hosp. Epidemiol. 37:1342-1348.

Magill, S. S., J. R. Edwards, W. Bamberg, Z. G. Beldavs, G. Dumyati, M. A. Kainer, R. Lynfield, R. et al. 2014. "Multistate point-prevalence survey of health care-associated infections." N. Engl. J. Med. 370:1198-1208.

Napolitano, L. M. and C. E. Edmiston Jr. 2017. “Clostridium difficile disease: Diagnosis, pathogenesis, and treatment update." Surgery 162:325-348.

Norén, T. 2010. "Clostridium difficile and the disease it causes." Methods Mol. Biol. 646:9-35.

Pagano, L., and M. Caira. 2012. "Risks for infection in patients with myelodysplasia and acute leukemia." Currn Opin Infect Dis. 25:612-618.

Pépin, J., L. Valiquette, and B. Cossette. 2005. "Mortality attributable to nosocomial Clostridium difficile-associated disease during an epidemic caused by a hypervirulent strain in Quebec." CMAJ. 173:1037-1042.

Rohlke, F. and N. Stollman. 2012. "Fecal microbiota transplantation in relapsing Clostridium difficile infection." Therap. Adv. Gastroenterol. 5:403-420.

Scott, R. D. II. 2009. “The direct medical costs of healthcare-associated infections in US hospitals and the benefits of prevention." Last accessed on November 6, 2017. http://www.cdc.gov/hai/pdfs/hai/scott_costpaper.pdf

Sehulster, L., and R. Y. W. Chinn. 2003. "Guidelines for environmental infection control in health-care facilities: recommendations of CDC and the Healthcare Infection
Control Practices Advisory Committee (HICPAC)." MMWR 52:1-42.

Siegel, J. D., E. Rhinehart, M. Jackson, L. Chiarello, and the Healthcare Infection Control Practices Advisory Committee. 2007. "2007 Guideline for isolation precautions: preventing transmission of infectious agents in healthcare settings." Last accessed on November 30, 2017. https:// www.cdc.gov/infectioncontrol/pdf/guidelines/isolationguidelines.pdf

Squellati, R. 2017. "Evidence-based practice in the treatment for antibiotic-associated diarrhea in the intensive care unit." Crit. Care Nurs. Clin. North Am. https://doi. org/10.1016/j.cnc.2017.10.008.

Steiner, C., M. Barrett, and Y. Sun. 2015. "HCUP Projections: Clostridium Difficile Hospitalizations 2004-2015. 2015. HCUP Projections Report \#2015-02." Last accessed on Nov 15, 2017. https://www.hcup-us.ahrq.gov/reports/ projections/2015-02.pdf

Stone, N. E., L. C. Sidak-Loftis, J. W. Sahl, A. J. Vazquez, K. B. Wiggins, J. D. Gillece et al. 2016. "More than 50\% of Clostridium difficile isolates from pet dogs in Flagstaff, USA, carry toxigenic genotypes." PLOS One 11:e0164504.

Teter, J, Florida Department of Health, Division of Disease Control and Health Protection. 2015. "Clostridium difficile diagnoses during hospitalizations, Florida, 2008-2013." Last accessed on November 28, 2017. http://www.floridahealth. gov/diseases-and-conditions/clostridium-difficile/_documents/clostridium-difficile-diagnoses-during-hospitalizations.pdf.

Tredget, E. E., H. A. Shankowsky, R. Rennie, R. E. Burrell, and S. Logsetty. 2004. "Pseudomonas infections in the thermally injured patient." Burns 30:3-26. 
Table 1. Estimated numbers of major types of healthcare-associated infection in the United States in $2011^{*}$.

\begin{tabular}{|l|c|}
\hline Type of Infection & Estimated No. \\
\hline Pneumonia & 157,500 \\
\hline Surgical site infections & 157,500 \\
\hline Gastrointestinal infections & 123,100 \\
\hline Urinary tract infections & 93,300 \\
\hline Primary bloodstream infections & 71,900 \\
\hline Other types of infections & 118,500 \\
\hline Estimated total number of healthcare-associated infections & $\mathbf{7 2 1 , 8 0 0}$ \\
\hline *Modified from the table published by Magill et al. (2014) and the CDC (2016a) & \\
\hline
\end{tabular}

Table 2. Reported top ten causative pathogens of 504 healthcare-associated infections in the United States acute care hospitals in $2011^{*}$.

\begin{tabular}{|l|c|}
\hline Type of Pathogen & $\%$ \\
\hline Clostridium difficile & 12.1 \\
\hline Staphylococcus aureus & 10.7 \\
\hline Klebsiella pneumoniae or K. oxytoca & 9.9 \\
\hline Escherichia coli & 9.3 \\
\hline Pseudomonas aeruginosa & 8.7 \\
\hline Candida species & 7.1 \\
\hline Urinary tract infections & 6.3 \\
\hline Streptococcus species & 5.0 \\
\hline Coagulase-negative Staphylococcus species & 4.8 \\
\hline Enterobacter species & 3.2 \\
\hline *Modified from the table published by Magill et al. (2014) & \\
\hline
\end{tabular}

